\title{
Adherence to the SEP-1 Sepsis Bundle in Hospital-Onset v. Community-Onset Sepsis: a Multicenter Retrospective Cohort Study
}

\author{
Jonathan D. Baghdadi, MD, $P h D^{7}$, Mitchell D. Wong, $M D, P h D^{2}$, \\ Daniel Z. Uslan, MD, MBA ${ }^{3}$, Douglas Bell, MD, PhD², William E. Cunningham, MD, $M P H^{4}$, \\ Jack Needleman, $P h D^{4}$, Russell Kerbel, $M D^{5}$, and Robert Brook, MD, SCD $5,6,7$
}

\begin{abstract}
'Department of Epidemiology and Public Health, University of Maryland School of Medicine, Baltimore, MD, USA; ${ }^{2}$ UCLA Division of General Internal Medicine, Los Angeles, CA, USA; ' ${ }^{3}$ CLA Division of Infectious Diseases, Los Angeles, CA, USA; ${ }^{4}$ UCLA Fielding School of Public Health, Department of Health Policy and Management, Los Angeles, CA, USA; ${ }^{\circ}$ UCLA Department of Medicine, Los Angeles, CA, USA; 'RAND Corporation, Santa Monica, CA, USA; ' ${ }^{D}$ avid Geffen School of Medicine at University of California, Los Angeles, Los Angeles, CA, USA.
\end{abstract}

BACKGROUND: Sepsis is the leading cause of in-hospital death. The SEP-1 sepsis bundle is a protocol for early sepsis care that requires providers to diagnose and treat sepsis quickly. Limited evidence suggests that adherence to the sepsis bundle is lower in cases of hospital-onset sepsis.

OBJECTIVE: To compare sepsis bundle adherence in hospital-onset vs. community-onset sepsis.

DESIGN: Retrospective cohort study using multivariable analysis of clinical data.

PARTICIPANTS: A total of 4658 inpatients age 18 or older were identified by diagnosis codes consistent with sepsis or disseminated infection.

SETTING: Four university hospitals in California between 2014 and 2016.

MAIN OUTCOMES AND MEASURES: The primary outcome was adherence to key components of the sepsis bundle defined by the Centers for Medicare and Medicaid Services in their core measure, SEP-1. Covariates included clinical characteristics related to the patient, infection, and pathogen.

KEY RESULTS: Compared with community-onset, cases of hospital-onset sepsis were less likely to receive SEP-1 adherent care (relative risk 0.33, 95\% confidence interval $0.29-0.38, p<0.001)$. With the exception of vasopressors (RR 1.11, $p=0.002$ ), each component of SEP-1 evaluated-blood cultures (RR 0.76, $p<0.001$ ), serum lactate (RR 0.51, $p<0001)$, broad-spectrum antibiotics (RR 0.62, $p<0.001$ ), intravenous fluids $(0.47, p<0.001)$, and follow-up lactate (RR 0.71, $p<0.001)$-was less likely to be performed within the recommended time frame in hospital-onset sepsis. Within the hospital, cases of hospital-onset sepsis arising on the ward were less likely to receive SEP-1-adherent care than were cases arising in the intensive care unit (RR 0.68, $p=0.004$ ).

Prior Presentations This work was previously presented at IDWeek 2018 from October 3 to 7 in San Francisco.

Electronic supplementary material The online version of this article (https://doi.org/10.1007/s11606-020-05653-0) contains supplementary material, which is available to authorized users.

Received March 25, 2019

Revised September 26, 2019

Accepted December 10, 2019

Published online February 10, 2020
CONCLUSIONS: Inpatients with hospital-onset sepsis receive different management than individuals with community-onset sepsis. It remains to be determined whether system-level factors, provider-level factors, or factors related to measurement explain the observed variation in care or whether variation in care affects outcomes.

KEYWORDS: health services research; performance measurement; critical care; infectious disease; hospital medicine.

J Gen Intern Med 35(4):1153-60

DOI: $10.1007 / \mathrm{s} 11606-020-05653-0$

(c) Society of General Internal Medicine 2020

\section{INTRODUCTION}

Sepsis is a leading cause of death in the hospital. ${ }^{1,2}$ Though the definition varies, 10-20\% of cases can be considered "hospital-onset," meaning the signs and symptoms of sepsis developed after hospital admission. ${ }^{3-5}$ Hospital-onset is associated with a mortality rate that is twice as high as communityonset. $^{4,6,7}$ It is unknown whether patients with hospital-onset sepsis receive the same quality of care as do patients with community-onset.

Protocols for early sepsis care, called "sepsis bundles," have been demonstrated to reduce mortality in community-onset sepsis when implemented in the emergency department (ED). ${ }^{8}, 9$ Though sepsis bundles do not have the same basis in evidence in hospital-onset sepsis, ${ }^{9-11}$ the Centers for Medicare and Medicaid Services (CMS) and professional societies recommend their use in all cases of sepsis with organ dysfunction, including those of hospital-onset. ${ }^{12-14}$

Overall adherence to the CMS sepsis bundle, known as SEP-1, is only $30-50 \% .^{10,15-17}$ We hypothesize that adherence may be lower in hospital-onset sepsis. Inpatient providers may be more likely to attribute signs of sepsis to other causes, such as postoperative fever, to remain anchored on admitting diagnoses, or to decide that a standardized care protocol is not appropriate for their patient. We sought to determine whether 
adherence to SEP-1 differs between community- and hospitalonset sepsis, and, by extension, whether the highest risk patients with sepsis are as likely to receive the standard of care.

\section{MATERIALS AND METHODS}

\section{Data Source}

We obtained clinical data from the electronic health records of four University of California hospitals offering diverse clinical services. All data were collected during routine clinical care. The UCLA IRB provided approval.

\section{Definitions}

We defined "sepsis" as suspected infection with organ dysfunction, including syndromes previously called severe sepsis and septic shock, based on Sepsis-3. ${ }^{18}$ Other definitions were based on the CMS core measure SEP-1 (National Quality Forum \#0500). ${ }^{14}$ "Time zero" of sepsis was determined using an automated algorithm (Appendix 1 in the Electronic Supplementary Material). To validate this process, time zero as identified by the automated algorithm was compared against the "time of presentation date" identified by trained chart abstracters in the subset of patients who underwent review for official SEP-1 reporting (Appendix 2 in the Electronic Supplementary Material). Another validation was performed comparing time zero from the automated algorithm against provider notes in a second subset with hospital-onset sepsis. Community-onset and hospital-onset were defined by time zero in the ED and on an inpatient unit, respectively.

\section{Variables}

The primary outcome was adherence to the SEP-1 sepsis bundle, an all-or-nothing measure requiring 4 components within $3 \mathrm{~h}$ of time zero and 2 components within $6 \mathrm{~h}$ of time zero. The 3-h components include (1) blood cultures prior to antibiotics, (2) broad-spectrum antibiotics, (3) a serum lactate, and, if the lactate is elevated or the blood pressure low, (4) intravenous crystalloid. Within $1 \mathrm{~h}$ of the fluid bolus completing, the patient's blood pressure must be checked twice to evaluate for persistent hypotension. The 6-h components are (5) a repeat lactate if initially abnormal and (6) vasopressors for persistent hypotension after intravenous fluids. Reassessment of tissue perfusion within $6 \mathrm{~h}$ of time zero, a seventh component, was excluded due to inherent flexibility and the lack of a pertinent field in the electronic health record. Rechecking the blood pressure was excluded due to inconsistent charting of fluid infusion rates. Secondary outcomes included each individual bundle component and the 3$\mathrm{h}$ components as a block (the 3 -h bundle).

Covariates included year of admission, age, gender, baseline health, pathogen, source of infection, immunosuppression, postoperative status, and hospital (see Appendix 3 in the Electronic Supplementary Material for details of variable construction). Baseline health was represented by count of conditions from the Elixhauser Comorbidity Index. ${ }^{19,}{ }^{20}$ Categories for source of infection were pneumonia, urinary tract infection, skin and/or soft tissue infection, and bloodstream infection (excluding possible skin contaminants, see Appendix 1 in the Electronic Supplementary Material).

\section{Study Design}

We conducted a retrospective study evaluating adherence to the SEP-1 bundle in community-onset and hospital-onset sepsis.

\section{Inclusion and Exclusion Criteria}

All encounters for individuals age 18 or older between October 1, 2014 and October 1, 2016 associated with a diagnosis of sepsis or disseminated infection were eligible (Appendix 4 in the Electronic Supplementary Material). Individual patients were able to contribute multiple encounters. Exclusion criteria were from SEP-1: hospitalization $>120$ days or $<6 \mathrm{~h}$, admission by acute care transfer, or receipt of intravenous antibiotics for $\geq 24 \mathrm{~h}$ at time zero.

For encounters prior to October 1, 2015, the set of International Classification of Diseases, Ninth Revision, Clinical Modification (ICD-9 CM) diagnosis codes used to identify sepsis encounters was based on methodology employed by Martin et al. ${ }^{21}$ that has since been validated and replicated. ${ }^{22-}$

${ }^{24}$ For hospitalizations beginning October 2015, ICD-10-CM diagnosis codes provided by CMS in SEP-1 were paired with diagnosis codes for organ dysfunction in a process analogous to the Martin methodology (Appendix 4 in the Electronic Supplementary Material).

\section{Statistical Analysis}

Multilevel mixed-effects Poisson regression with robust error variance was used to evaluate the relative risk of adherence since relative risk is the parameter of interest. ${ }^{25}$ Fixed and random effects were incorporated to account for clustering by and within hospital units. Survival analysis, including Kaplan-Meier curves and Cox proportional hazards modeling, evaluated the association between hospital-onset of sepsis and time to 3-h bundle components. Analyses were performed using Stata/IC version 14.1.

\section{RESULTS}

A total of 4658 patient encounters were analyzed (Fig. 1). Characteristics of the sample are described in Table 1 (see Supplemental Table 4 for differences by hospital). The average age was $63,44 \%$ were female, and the median number of Elixhauser comorbidities was 5. A total of 1437 individuals (30.9\%) received the SEP-1 bundle within the recommended time frame. Patients with hospital-onset were younger (average age 60.9 v. 64.4 years), more immunosuppressed (37.6\% v. $26.4 \%)$, and more often postoperative $(15.9 \%$ vs. $2.5 \%)$. 


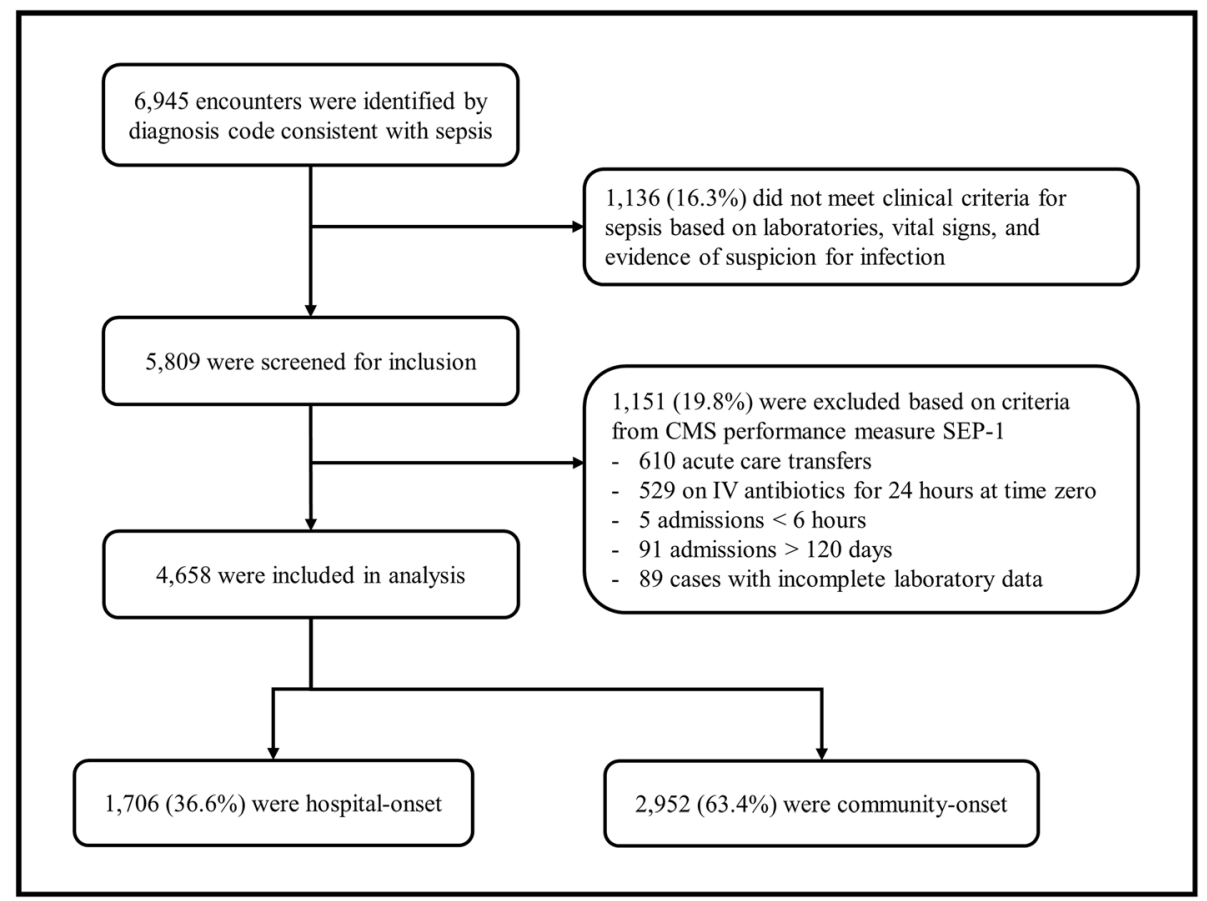

Figure 1 Patient flow diagram. Legend: CMS, The Centers for Medicare \& Medicaid Services. SEP-1, Early Management Bundle, Severe Sepsis / Septic Shock.

Community-onset sepsis was more commonly associated with bacteremia (33.2\% vs. $22.0 \%)$ or MRSA (10.8\% v. $8.7 \%)$.

Multivariable regression demonstrated factors associated with timely administration of SEP-1 (Table 2). Cases of hospital-onset sepsis were less likely to receive SEP-1adherent care $39.9 \%$ probability for community-onset, $13.0 \%$ for hospital-onset; RR $0.33, p<0.001)$. SEP-1 nonadherence was also associated with postoperative status (RR $0.59, p<0.001)$ and increased number of Elixhauser comorbidities (RR 0.98 for each additional comorbidity, $p<0.001$ ). The presence of fever (RR 1.37, $p<0.001$ ) or bacteremia (RR $1.20, p<0.001)$ were associated with SEP-1 adherence. These associations held when excluding the SEP-1 requirement for intravenous fluids. On stratified analyses, hospital-onset was a significant predictor of non-adherence at all four hospitals and regardless of admitting provider specialty (Supplemental Tables 5 and 6). In a sensitivity analysis, time zero $>48 \mathrm{~h}$ after arrival was evaluated as an alternative definition of hospitalonset and demonstrated a similar relationship with likelihood of adherence as the definition used elsewhere in this study (RR $0.28, p<0.001)$.

Adherence to SEP-1 differed across inpatient areas (Table 3). Compared with the ward, cases of sepsis arising in the intensive care unit (ICU) were more likely to receive the complete SEP-1 bundle (RR $1.48, p=0.004$ ) and more likely to have a serum lactate checked (RR 1.42, $p<0.001)$. When indicated, cases in the ICU were less likely to receive intravenous fluids (RR $0.69, p=0.013$ ) but more likely to be started on vasopressors (RR 1.66, $p<0.001)$. Cases arising in the perioperative area were less likely than those on the ward to have timely blood cultures ( RR $0.75, p=0.002)$.
Time-to-event analysis was performed for the 3-h bundle components (Fig. 2A-D). The median time from onset of sepsis to completion of the 3 -h bundle was $3.0 \mathrm{~h}$ (95\% confidence interval, 2.5-3.7) in community-onset and $79.6 \mathrm{~h}(95 \%$ confidence interval, 67.5-96.8) in hospital-onset. Median time to serum lactate $(0.0 \mathrm{~h}$ in community-onset, $5.9 \mathrm{~h}$ in hospitalonset) and intravenous fluids (1.8 h in community-onset, $19.3 \mathrm{~h}$ in hospital-onset) were significantly longer in hospital-onset ( $p<0.001$ by log-rank test comparing survival curves). On the other hand, median time of blood cultures and broad-spectrum antibiotics coincided with or preceded time zero in both community-onset and hospital-onset. Cox proportional hazards models identified that patients with hospitalonset sepsis were less likely to have timely blood cultures (HR $0.66, p<0.001)$, serum lactate $(0.47, p<0.001)$, broadspectrum antibiotics (HR $0.65, p<0.001$ ), intravenous fluids (HR 0.60, $p<0.001$ ), or the 3 -h bundle (HR 0.43, $p<0.001$ ).

\section{DISCUSSION}

The SEP-1 bundle, which is embodied in professional society guidelines and has been adopted by CMS as a core measure, recommends that all cases of sepsis be managed the same way. However, in this multicenter cohort study, providers appeared to approach hospital-onset and community-onset sepsis differently. In our sample, only $12.7 \%$ of hospital-onset cases received SEP-1-adherent care, compared with $45.9 \%$ of community-onset cases. Given that the attributable mortality is higher in hospital-onset sepsis than community-onset, less 
Table 1 Characteristics of Patients with Community-Onset v. Hospital-Onset Sepsis

\begin{tabular}{|c|c|c|c|}
\hline & Community-onset $(n=2952)$ & Hospital-onset $(n=1706)$ & $p$ value \\
\hline Age - mean $(\mathrm{sd})$ & $64.4(17.9)$ & $60.9(17.8)$ & $<0.001 \dagger$ \\
\hline Female, $n(\%)$ & 1307 (44.3) & $755(44.3)$ & 0.99 \\
\hline $\mathrm{ECI}$-median (IQR) & $5(3-7)$ & $5(3-7)$ & $0.11 \ddagger$ \\
\hline Immunosuppressed, $n(\%)$ & $780(26.4)$ & $641(37.6)$ & $<0.001$ \\
\hline Postoperative, $n(\%)$ & $73(2.47)$ & $271(15.9)$ & $<0.001$ \\
\hline \multicolumn{4}{|l|}{ Infection site } \\
\hline Pneumonia, $n(\%)$ & $1410(47.8)$ & $813(47.7)$ & 0.94 \\
\hline UTI, $n(\%)$ & $1055(35.7)$ & $555(32.5)$ & 0.027 \\
\hline SSTI, $n(\%)$ & 327 (11.1) & 195 (11.4) & 0.71 \\
\hline BSI, $n(\%)$ & $979(33.2)$ & $375(22.0)$ & $<0.001$ \\
\hline Multiple, $n(\%)$ & $1069(36.2)$ & $481(28.2)$ & $<0.001$ \\
\hline \multicolumn{4}{|l|}{ Pathogen } \\
\hline MSSA, $n(\%)$ & $183(6.20)$ & $115(6.74)$ & 0.47 \\
\hline MRSA, $n(\%)$ & $318(10.8)$ & $149(8.73)$ & 0.026 \\
\hline MDR Gram-negative, $n(\%)$ & $151(5.12)$ & $100(5.86)$ & 0.28 \\
\hline VRE, $n(\%)$ & $58(1.96)$ & $40(2.34)$ & 0.38 \\
\hline \multicolumn{4}{|l|}{ Organ dysfunction at time 0} \\
\hline Low blood pressure, $n(\%)$ & 1398 (47.4) & $965(56.6)$ & $<0.001$ \\
\hline Respiratory failure, $n(\%)$ & $250(8.47)$ & $225(13.2)$ & $<0.001$ \\
\hline Elevated creatinine, $n(\%)$ & $359(12.2)$ & $186(10.9)$ & 0.20 \\
\hline Elevated bilirubin, $n(\%)$ & $189(6.40)$ & $143(8.38)$ & 0.011 \\
\hline Thrombocytopenia, $n(\%)$ & $300(10.2)$ & $200(11.7)$ & 0.097 \\
\hline Coagulopathy, $n(\%)$ & $276(9.35)$ & $248(14.5)$ & $<0.001$ \\
\hline Elevated serum lactate, $n(\%)$ & $694(23.5)$ & $118(6.92)$ & $<0.001$ \\
\hline Multiple, $n(\%)$ & $368(12.5)$ & $264(15.5)$ & 0.003 \\
\hline \multicolumn{4}{|l|}{ Outcome } \\
\hline LOS-median (IQR) & $8(4.3-15.5)$ & $15.5(8-27.5)$ & $<0.001 \ddagger$ \\
\hline Mortality, $n(\%)$ & $553(18.7)$ & $368(21.6)$ & 0.019 \\
\hline
\end{tabular}

ECI, Elixhauser Comorbidity Index; UTI, urinary tract infection; SSTI, skin/soft tissue infection; BSI, bloodstream infection; MSSA, Methicillin-sensitive Staphylococcus aureus; MRSA, methicillin-resistant Staphylococcus aureus; MDR, multi-drug resistant; VRE, vancomycin-resistant enterococci; LOS, overall length of stay for admission. *All p values from chi-square test of proportions unless otherwise specified; $\dagger$ Student's $t$ test; $\neq$ Rank sum test

frequent guideline adherence in this population requires further exploration.

EDs are designed to triage and deliver rapid care for conditions in which every minute counts, such as myocardial infarction or stroke. Thus, it is perhaps unsurprising that SEP-1 adherence was higher in the ED than in the hospital, a monitored setting. Independent of patient characteristics, cases of hospital-onset sepsis were 3-times less likely (risk ratio 0.33 , risk difference 0.27 ) to receive guideline-adherent care. This association was observed at every study site and has been reported by others, suggesting a relationship that is systematic to inpatient care (though notably, only delayed antibiotics have been associated with increased mortality in hospital-onset sepsis). ${ }^{10,26}$

The hospital is a complex system, ${ }^{27,28}$ and differences in systems of care delivery between the ED and inpatient areas likely contribute to the observed variation in sepsis management. The relevant factors are structural (patient-to-staff ratios, colocation of providers and patients), functional (rounding schedules, team size, frequency of communication), and related to hospital policy (where vasopressors can be administered, whether "code sepsis" can be called). Though similar, even the ICU and ED differ in ways that may affect their ability to mount a rapid response, such as the presence of an attending overnight or whether an elevator ride is required when traveling to the computed tomography scanner. Each area has evolved to match the expected length of stay: hours in the ED vs. days in the inpatient areas. The timescale in the ED is a better fit for SEP-1.
Provider-level differences between the ED and inpatient areas likely also contribute to the observed variation in sepsis care. Context affects clinical reasoning, ${ }^{29,30}$ and the additional information available to inpatient providers may make them more susceptible to cognitive biases such as anchoring. To illustrate this point, consider the steps required for early sepsis care. Emergency providers assess an undifferentiated patient and, if sepsis is recognized, respond accordingly. For inpatient providers, onset of sepsis is more likely to represent a change. The inpatient provider must make an assessment, filter new information, and integrate it with what is already known. If sepsis is recognized, the physician must pivot from the admitting diagnosis to enact a new plan of care. The question is not whether providers can respond, but whether they are as likely to respond within $3 \mathrm{~h}$. Communication is critical, and delays may be amplified if coordination is required among multiple providers, such as consultants or trainees. ${ }^{31}$

Alternatively, because more information about the patient is available in hospital-onset sepsis, inpatient providers may feel more confident observing while withholding components of SEP-1. To determine whether providers were selecting among bundle components, we evaluated reconfigured bundles that omitted intravenous fluids and serum lactate (Table 2). Regardless of bundle configuration, individuals with hospitalonset sepsis were less likely to receive timely care. Thus, low SEP-1 adherence in hospital-onset sepsis appears unrelated to providers' doubts regarding the hemodynamic components. ${ }^{32}$ 
Table 2 Association Between Sepsis Bundle and Patient Factors in Terms of Relative Risk

\begin{tabular}{llll}
\hline \hline & Sepsis bundle & Sepsis bundle without IV fluids & Blood cultures and antibiotics only \\
\hline Age (in 10-year increments) & $1.02(p=0.10)$ & $1.03(p=0.003)$ & $1.00(p=0.22)$ \\
Female & $1.00(p=0.95)$ & $0.99(p=0.85)$ & $1.01(p=0.72)$ \\
ECI & $0.98(p<0.001)$ & $0.98(p=0.002)$ & $1.00(p=0.27)$ \\
Immunosuppressed & $0.97(p=0.55)$ & $1.03(p=0.39)$ & $1.04(p=0.11)$ \\
Postoperative & $0.59(p<0.001)$ & $0.66(p<0.001)$ & $0.63(p<0.001)$ \\
Fever & $1.37(p<0.001)$ & $1.34(p<0.001)$ & $1.31(p<0.001)$ \\
Hospital-onset of sepsis & $0.33(p<0.001)$ & $0.37(p<0.001)$ & $0.61(p<0.001)$ \\
Infection site & & & $1.06(p=0.002)$ \\
Pneumonia & $1.04(p=0.38)$ & $1.03(p=0.44)$ & $0.99(p=0.73)$ \\
UTI & $0.99(p=0.74)$ & $0.98(p=0.49)$ & $1.06(p=0.060)$ \\
SSTI & $1.10(p=0.13)$ & $1.14(p=0.007)$ & $1.11(p<0.001)$ \\
BSI & $1.22(p<0.001)$ & $1.15(p<0.001)$ & $0.93(p=0.048)$ \\
Pathogen & & & $1.05(p=0.074)$ \\
MSSA & $0.97(p=0.71)$ & $0.97(p=0.57)$ & $1.08(p=0.052)$ \\
MRSA & $1.05(p=0.45)$ & $1.06(p=0.23)$ & $1.02(p=0.78)$ \\
MDR Gram-negative & $1.00(p=0.96)$ & $1.01(p=0.93)$ &
\end{tabular}

Italics indicate significance at the level of $\alpha<0.05$

ECI, Elixhauser Comorbidity Index; UTI, urinary tract infection; SSTI, skin/soft tissue infection; BSI, bloodstream infection; MSSA, methicillin-sensitive Staphylococcus aureus; MRSA, methicillin-resistant Staphylococcus aureus; MDR, multi-drug resistant; VRE, vancomycin-resistant enterococci

However, providers may have other reasons for withholding treatment bundles in hospital-onset sepsis, such as terminal illness. $^{33-37}$

Finally, lower adherence to SEP-1 in hospital-onset sepsis may be related to less precision when identifying time zero in this population. Based on the validation of time zero in our data set (see Appendix 2 in the Electronic Supplementary Material), we suspect that time zero can be ascertained more precisely in community-onset sepsis, when there are fewer laboratory values and vital signs obtained before sepsis manifests, than in hospital-onset sepsis, when days may pass before labs, vital signs, and clinical documentation meet the criteria. If estimation of time zero has higher variance in hospital-onset than in community-onset sepsis (as may occur if labs are being checked less frequently in the hospital than in the ED), then more hospital-onset cases may be misclassified as non-adherent or meeting the measure's exclusion criteria. Moreover, if the confidence intervals surrounding time zero in hospital-onset sepsis exceed $3 \mathrm{~h}$, then whether bundle components were performed within $3 \mathrm{~h}$ cannot be determined at all. The precision of time zero measurement in hospital-onset sepsis must be characterized to determine the validity of SEP-1 in this population.

Beyond issues related to measurement, our findings illustrate issues with SEP-1 as a performance measure. SEP-1 adherence varies dramatically based on patient-level characteristics. If the intended purpose of SEP-1 is hospital comparison, adjustment will be needed to account for hospital-level differences in the patient populations served. Otherwise, facilities that cater to groups in whom the likelihood of adherence is low, such as postoperative patients, will be systematically underrated.

It remains to be determined if better adherence to SEP-1 would improve outcomes from hospital-onset sepsis. In a previous study, adherence was not found to be associated with a mortality benefit. ${ }^{10}$ However, sepsis is a heterogeneous syndrome, ${ }^{38,39}$ and subtypes of sepsis may respond differently to treatment. ${ }^{26}$ In our sample, fever was a strong predictor of SEP-1 adherence, even in patients in whom fever may be considered an unreliable indicator of infection, such as those who were postoperative (RR 2.11, 95\% CI 1.13-3.91) or immunosuppressed (RR 1.47, 95\% CI 1.26-1.72). Future research into the impact of sepsis bundles should consider their effectiveness in the subgroups who are most likely to receive them, such as patients with fever, compared with patients who are more likely to present with atypical signs and symptoms of infection.

\section{Limitations}

We did not examine outcomes such as mortality, morbidity, or length of stay. Instead, the primary outcome was adherence to SEP-1, the current standard of care. ${ }^{40}$ We acknowledge that the quality of evidence supporting SEP-1 in hospital-onset sepsis is poor, that SEP-1 encourages liberal antibiotic use, and that a proportion of sepsis mortality may not be preventable. ${ }^{32,41}$ As the consensus regarding optimal management of sepsis evolves, measures that better reflect quality may emerge. Further, we caution that implementation of SEP-1 must be balanced against policies and procedures for antimicrobial stewardship. Future studies evaluating the clinical impact of SEP-1 should incorporate antibiotic use as a balancing measure.

Our sample may not generalize to patients with sepsis in other hospitals and regions. Because we identified patients using diagnosis codes, we were unable to capture individuals in whom sepsis and organ dysfunction were never diagnosed or documented/coded in the electronic health record. This methodology has been validated and mirrors the process used with SEP-1. However, because of limitations of the sample, our conclusions should only apply to the individuals covered by SEP-1, rather than the broader population with sepsis. 
Table 3 Relative Risk of SEP-1 Sepsis Bundle Components in Hospital-Onset Compared to Community-Onset Sepsis

\begin{tabular}{|c|c|c|c|c|}
\hline & Hospital-onset & $\overline{\text { ICU-onset } \dagger}$ & Ward-onset $\dagger$ & Perioperative $\dagger$ \\
\hline Blood cultures & $0.76(p<0.001)$ & $0.78(p<0.001)$ & $0.77(p<0.001)$ & $0.58(p<0.001)$ \\
\hline Serum lactate & $0.51(p<0.001)$ & $0.61(p<0.001)$ & $0.43(p<0.001)$ & $0.39(p<0.001)$ \\
\hline Broad antibiotics & $0.62(p<0.001)$ & $0.66(p<0.001)$ & $0.60(p<0.001)$ & $0.49(p<0.001)$ \\
\hline IV fluids* & $0.47(p<0.001)$ & $0.41(p<0.001)$ & $0.59(p<0.001)$ & $0.44(p=0.001)$ \\
\hline Follow-up lactate* & $0.71(p<0.001)$ & $0.76(p=0.005)$ & $0.66(p<0.001)$ & $0.77(p=0.22)$ \\
\hline Vasopressors* & $1.11(p=0.002)$ & $1.26(p<0.001)$ & $0.76(p=0.001)$ & $0.95(p=0.65)$ \\
\hline 3-hour block & $0.31(p<0.001)$ & $0.35(p<0.001)$ & $0.29(p<0.001)$ & $0.23(p<0.001)$ \\
\hline 6-hour block* & $0.97(p=0.39)$ & $1.17(p<0.001)$ & $0.66(p<0.001)$ & $0.83(p=0.17)$ \\
\hline Complete bundle & $0.33(p<0.001)$ & $0.41(p<0.001)$ & $0.28(p<0.001)$ & $0.21(p<0.001)$ \\
\hline
\end{tabular}

*If required. $\uparrow$ Relative to the Emergency Department. ICU, intensive care unit

Temporal trends in the incidence of sepsis suggest that coding practices have changed. ${ }^{4,6}$ Consequently, our results may be susceptible to bias from local interventions to change coding over time. Comparability between the communityonset and hospital-onset cohorts may be affected by differences in coding between the emergency and inpatient areas (i.e., coding in the inpatient area depends on problem lists generated by house staff without training in medical billing). Confounding may occur if entry of a diagnosis code is linked to treatment (i.e., ED staff only code "sepsis" if the sepsis protocol was initiated).
We determined adherence using laboratory, vital sign, and medication data extracted from the electronic health record, rather than manual chart review. Thus, we likely ascertained adherence incorrectly in cases with an administrative contraindication (i.e., provider documented patient refusal) or when time zero might hinge on documentation (i.e., labs and vitals meet the criteria but provider documents "this is not sepsis"). Given that chart review for SEP-1 is known to be an imperfect process with poor interobserver reliability, use of non-standard methodology was considered acceptable to allow evaluation of
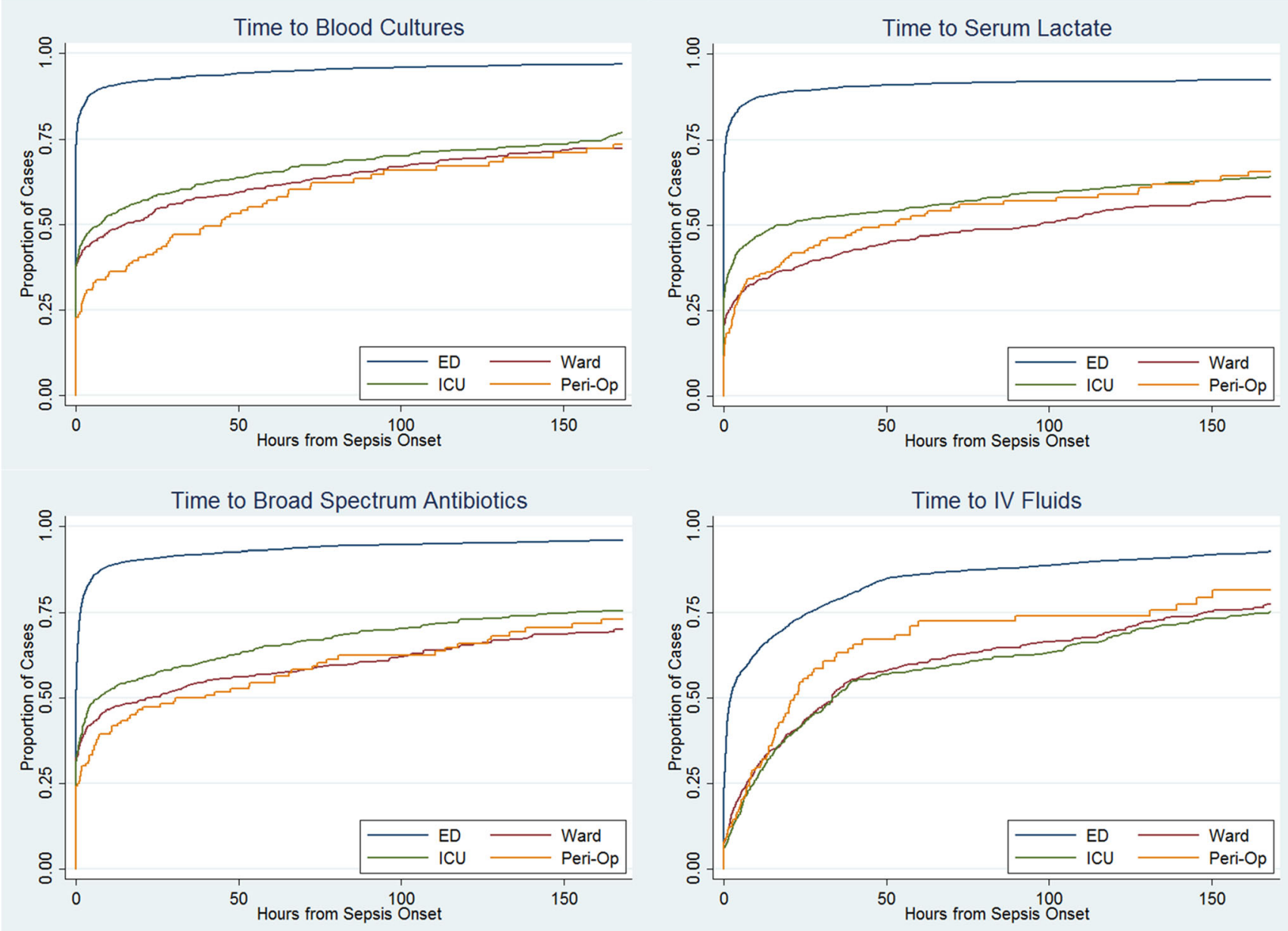

Figure 2 Parts A-D. Time to 3-hour sepsis bundle and components. Legend: Curves have been truncated at 7 days. ED, emergency department. ICU, intensive care unit. Peri-op, perioperative area. IV, intravenous. 
a larger sample. ${ }^{42-44}$ The omission of re-evaluation of tissue perfusion, one of the seven components of SEP-1, may bias estimates of overall SEP-1 adherence but should not affect the relationships between predictors and timely administration of core bundle components.

To isolate inpatient processes of care, we defined hospitalonset of sepsis by time zero after arrival on an inpatient unit. This definition may limit comparability of this study to others that defined hospital-onset differently. ${ }^{5,10,}{ }^{44}$ However, a sensitivity analysis in which hospital-onset was defined by time zero $>48 \mathrm{~h}$ after admission did not change our findings.

During the study period, two of the four participating hospitals introduced nurse-initiated sepsis screening. Nurseinitiated sepsis screening has been associated with guideline adherence in sepsis, particularly in the ED. ${ }^{34,}{ }^{35}$ However, hospital staff have reported to us that implementation of screening was inconsistent. Thus, the impact of these initiatives on our results is not clear. If nurse-initiated sepsis screening affected SEP-1 adherence, we suspect it most likely occurred in the ICU, where patient-to-nurse ratios are low, or in the ED, where evidence supports nurse-initiated screening.

\section{CONCLUSIONS}

Though CMS applies the SEP-1 core measure uniformly to all patients with sepsis, providers appear to manage patients with hospital-onset and community-onset sepsis differently. While lower levels of adherence to SEP-1 in cases of hospital-onset sepsis may represent a quality gap, there are alternative reasons for the observed differences in care, including potential measurement error. Further, it remains to be determined how adherence to the SEP-1 bundle affects outcomes from hospital-onset sepsis. Consistent and adequate sepsis care will not be possible until the unique challenges related to hospitalonset sepsis, including issues in measurement of time zero, are better understood. Then, consideration should be given to whether systems of care delivery and protocols for early sepsis care can be redesigned to better suit this high-risk patient population.

Contributors: We thank Summer Gupta Maxwell, RN, MSN for her advice and insights while performing this work.

Corresponding Author: Jonathan D. Baghdadi, MD, PhD; Department of Epidemiology and Public Health University of Maryland School of Medicine, Baltimore, MD, USA (e-mail: jbaghdadi@som.umaryland. edu).

Funders We thank the UCLA Specialty Training and Advanced Research (STAR) Program for supporting and facilitating research by fellows in training. While performing this work, Dr. Baghdadi was supported by the Health Resources and Services Administration (HRSA) via a Ruth $L$. Kirschstein Institutional National Research Service Award (NRSA), grant number for Primary Care T32 (T32HP19001), and by the Veterans Administration Quality Scholars (VABS) program. The research described was further supported by NIH/National Center for Advancing Translational Science (NCATS)
UCLA Clinical and Translational Science Institute Grant Number UL1TROO1881.

\section{Compliance with Ethical Standards:}

Conflict of Interest: The authors declare that they do not have a conflict of interest.

\section{REFERENCES}

1. Mayr, F.B., S. Yende, and D.C. Angus, Epidemiology of severe sepsis. Virulence, 2014. 5(1): p. 4-11.

2. Elixhauser, A., Friedman, B, Stranges, E. Septicemia in U.S. Hospitals, 2009. Healthcare Cost and Utilization Project, Statical Brief \#122. 2011 [December 6, 2019]; Available from: http://www.hcup-us.ahrq.gov/ reports/statbriefs/sb122.pdf.

3. Novosad, S.A., Vital signs: epidemiology of sepsis: prevalence of health care factors and opportunities for prevention. MMWR. Morbidity and mortality weekly report, 2016. 65.

4. Rhee, C., et al., Incidence and Trends of Sepsis in US Hospitals Using Clinical vs Claims Data, 2009-2014. Jama, 2017. 318(13): p. 1241-1249.

5. Jones, S.L., et al., Outcomes and Resource Use of Sepsis-associated Stays by Presence on Admission, Severity, and Hospital Type. Med Care, 2016. 54(3): p. 303-10.

6. Gohil, S.K., et al., Impact of Policies on the Rise in Sepsis Incidence, 2000 2010. Clin Infect Dis, 2016. 62(6): p. 695-703.

7. Paoli, C.J., et al., Epidemiology and Costs of Sepsis in the United StatesAn Analysis Based on Timing of Diagnosis and Severity Level. Critical care medicine, 2018. 46(12): p. 1889-1897.

8. Seymour, C.w., et al., Time to Treatment and Mortality during Mandated Emergency Care for Sepsis. New England Journal of Medicine, 2017. 376(23): p. 2235-2244.

9. Odden, A.J., et al., A Systematic Assessment of the Surviving Sepsis Campaign's Evidence Supporting the Care of Patients with Severe Sepsis on the Wards. Ann Am Thorac Soc, 2015. 12(6): p. 956-8.

10. Rhee, C., et al., Compliance With the National SEP-1 Quality Measure and Association With Sepsis Outcomes: A Multicenter Retrospective Cohort Study. Crit Care Med, 2018. 46(10): p. 1585-1591.

11. Milano, P.K., et al., Sepsis Bundle Adherence Is Associated with Improved Survival in Severe Sepsis or Septic Shock. West J Emerg Med, 2018. 19(5): p. 774-781.

12. Dellinger, R.P., et al., Surviving sepsis campaign: international guidelines for management of severe sepsis and septic shock: 2012. Crit Care Med, 2013. 41(2): p. 580-637.

13. Commission, J., Specifications manual for national hospital inpatient quality measures. Version 5.2, 2017.

14. QualityNet: Inpatient Hospitals Specifications Manual, version 5.2. November 1, 2016 [Accessed December 6, 2019]; Available from: http:// www.qualitynet.org.

15. Venkatesh, A.K., et al., Preliminary Performance on the New CMS Sepsis 1 National Quality Measure: Early Insights From the Emergency Quality Network (E-QUAL). Ann Emerg Med, 2017.

16. Barochia, A.V., X. Cui, and P.Q. Eichacker, The Surviving Sepsis Campaign's Revised Sepsis Bundles. Current infectious disease reports, 2013. 15(5): p. https://doi.org/10.1007/s11908-013-0351-3.

17. Rhodes, A., et al., The Surviving Sepsis Campaign bundles and outcome: results from the International Multicentre Prevalence Study on Sepsis (the IMPreSS study). Intensive Care Med, 2015. 41(9): p. 1620-8.

18. Singer, M., et al., The Third International Consensus Definitions for Sepsis and Septic Shock (Sepsis-3). JAMA, 2016. 315.

19. Elixhauser, A., et al., Comorbidity measures for use with administrative data. Medical care, 1998. 36(1): p. 8-27.

20. Moore, B.J., et al., Identifying Increased Risk of Readmission and Inhospital Mortality Using Hospital Administrative Data: The AHRQ Elixhauser Comorbidity Index. Med Care, 2017. 55(7): p. 698-705.

21. Martin, G.S., et al., The epidemiology of sepsis in the United States from 1979 through 2000. New England Journal of Medicine, 2003. 348(16): p. 1546-1554.

22. Lagu, T., et al., What is the best method for estimating the burden of severe sepsis in the United States? J Crit Care, 2012. 27(4): p. 414.e1-9.

23. Kumar, G., et al., Nationwide trends of severe sepsis in the 21st century (2000-2007). Chest, 2011. 140(5): p. 1223-1231. 
24. Stoller, J., et al., Epidemiology of severe sepsis: 2008-2012. J Crit Care, 2015.

25. Zou, G., A Modified Poisson Regression Approach to Prospective Studies with Binary Data. American Journal of Epidemiology, 2004. 159(7): p. 702-706.

26. Seymour, C.w., et al., Derivation, Validation, and Potential Treatment Implications of Novel Clinical Phenotypes for Sepsis. JAMA, 2019. 321(20): p. 2003-2017.

27. Mahajan, A., et al., A hospital is not just a factory, but a complex adaptive system-implications for perioperative care. Anesthesia \& Analgesia, 2017. 125(1): p. 333-341.

28. Nugus, P., et al., Integrated care in the emergency department: A complex adaptive systems perspective. Social Science \& Medicine, 2010. 71(11): p. 1997-2004.

29. Durning, S.J., et al., The impact of selected contextual factors on experts' clinical reasoning performance (does context impact clinical reasoning performance in experts?). Advances in Health Sciences Education, 2012. 17(1): p. 65-79.

30. McBee, E., et al., Consequences of contextual factors on clinical reasoning in resident physicians. Adv Health Sci Educ Theory Pract, 2015. 20(5): p. 1225-36.

31. Rosenbaum, L., Divided We Fall. New England Journal of Medicine, 2019. 380(7): p. 684-688

32. Pepper, D.J., et al., Evidence Underpinning the Centers for Medicare \& Medicaid Services' Severe Sepsis and Septic Shock Management Bundle (SEP-1): A Systematic Review. Ann Intern Med, 2018. 168(8): p. 558-568.

33. Miguel, N., et al., Sepsis-related organ failure assessment and withhold ing or withdrawing life support from critically ill patients. Critical care (London, England), 1998. 2(2): p. 61-66.

34. Torsvik, M., et al., Early identification of sepsis in hospital inpatients by ward nurses increases 30-day survival. Critical Care, 2016. 20(1): p. 244.

35. Bruce, H.R., et al., Impact of nurse-initiated ED sepsis protocol on compliance with sepsis bundles, time to initial antibiotic administration, and in-hospital mortality. Journal of Emergency Nursing, 2015. 41(2): p. 130-137.
36. Sochalski, J., Is More Better?: The Relationship Between Nurse Staffing and the Quality of Nursing Care in Hospitals. Medical Care, 2004. 42(2): p. II-67-II-73.

37. Lucero, R.J., E.T. Lake, and L.H. Aiken, Variations in nursing care quality across hospitals. Journal of advanced nursing, 2009. 65(11): p. 2299-2310.

38. Shankar-Hari, M., D.A. Harrison, and K.M. Rowan, Differences in Impact of Definitional Elements on Mortality Precludes International Comparisons of Sepsis Epidemiology-A Cohort Study Illustrating the Need for Standardized Reporting. Crit Care Med, 2016. 44(12): p. 2223-2230.

39. Kempker, J.A. and G.S. Martin, Does Sepsis Case Mix Heterogeneity Prevent Outcome Comparisons? Critical care medicine, 2016. 44(12): p. 2288-2289.

40. Rhodes, A., et al., Surviving Sepsis Campaign: International Guidelines for Management of Sepsis and Septic Shock: 2016. Intensive Care Medicine, 2017. 43(3): p. 304-377.

41. Rhee, C., et al., Prevalence, Underlying Causes, and Preventability of Sepsis-Associated Mortality. JAMA Network Open, 2019. 2(2): p. e187571-e187571.

42. Aaronson, E.L., et al., New Mandated Centers for Medicare and Medicaid Services Requirements for Sepsis Reporting: Caution from the Field. J Emerg Med, 2017. 52(1): p. 109-116.

43. Gonet, J., et al., Interrate Reliability for SEP-1 Abstraction in a Multihospital Medical Center. Critical Care Medicine, 2016. 44(12): p. 96.

44. Rhee, C., et al., Variability in determining sepsis time zero and bundle compliance rates for the centers for medicare and medicaid services SEP-1 measure. Infection Control \& Hospital Epidemiology, 2018. 39(8): p. 994996.

Publisher's Note Springer Nature remains neutral with regard to jurisdictional claims in published maps and institutional affiliations. 